\title{
OPINIÃO DOS ESTUDANTES DE ENFERMAGEM SOBRE SAÚDE, ESPIRITUALIDADE E RELIGIOSIDADE
}

Daniele Corcioli Mendes ESPINHA a , Stéphanie Marques de CAMARGOb ${ }^{b}$ Sabrina Piccinelli Zanchettin SILVA Shirlene PAVELQUEIRES ${ }^{\mathrm{d}}$, Giancarlo LUCCHETTI ${ }^{\mathrm{e}}$

\section{RESUMO}

O presente estudo objetivou identificar as opiniões dos estudantes de Enfermagem em relação à interface entre saúde, espiritualidade e religiosidade e as informações fornecidas durante o período da formação acadêmica. Estudo transversal em que foram entrevistados 120 alunos do curso de enfermagem (82,1\%) de uma faculdade do estado de São Paulo durante maio e outubro de 2011 . Destes, $76 \%$ acreditam que a espiritualidade influencia na saúde. Apenas $10 \%$ consideram-se muito preparados para abordar aspectos espirituais dos pacientes e $54 \%$ apontaram que a formação universitária não oferece informações suficientes para desenvolver essa competência. O medo de impor pontos de vista religiosos aos pacientes é a principal barreira relacionada ao assunto. A maioria dos estudantes, 83\%, apontou que temas referentes a saúde e espiritualidade deveriam fazer parte dos currículos de Enfermagem. É necessária a implementação de cenários de aprendizagem durante a formação acadêmica que auxiliem o estudante a realizar o cuidado espiritual.

Descritores: Espiritualidade. Religião. Enfermagem. Ensino.

\section{RESUMEN}

Este estudio tuvo como objetivo identificar los puntos de vista de los estudiantes de enfermería relación con la interfaz entre la salud, la espiritualidad y la religiosidad y la información proporcionada durante el período de formación académica. Estudio transversal entrevistó a 120 estudiantes de enfermería (82,1\%) de una universidad en el estado de São Paulo entre mayo y octubre de 2011. De estos, el 76\% cree que la espiritualidad influye en la salud. Sin embargo, solo el 10\% se consideran muy preparados para hacer frente a los aspectos espirituales de los pacientes y el $54 \%$ indicó que la educación universitaria no proporciona información suficiente para esta competencia. El temor a la imposición de puntos de vista religiosos es la principal barrera sobre el tema. La mayoría de los estudiantes (el 83\%) indicó que las cuestiones relativas a salud y espiritualidad deben formar parte del currículo de enfermería. Es necesario implementar escenarios de aprendizaje durante su formación académica para ayudar a los estudiantes en el cuidado espiritual.

Descriptores: Espiritualidad. Religión. Enfermería. Enseñanza.

Titulo: Opiniones de los estudiantes de enfermería sobre la salud, la espiritualidad y la religiosidad.

\section{ABSTRACT}

The present study aimed to identify the opinions of nursing students in relation to the interface between health, spirituality and religiosity and the information provided during their undergraduate formation. A cross-sectional study was conducted in 2011 (May to October) through interviews on 120 students from a nursing school (82.1\%) located at São Paulo State, Brazil. From these, $76 \%$ believe that spirituality have an influence on health. However, only $10 \%$ consider themselves very prepared to address patients' spiritual aspects and 54\% stated that university education does not provide enough information to develop this competence. The fear of imposing religious beliefs is the main barrier related to this issue. Most students (83\%) indicated that issues related to health and spirituality should be part of the nursing curricula. Therefore, it's necessary to implement learning scenarios during their academic training to help students in spiritual care.

Descriptors: Spirituality. Religion. Nursing. Teaching.

Title: Nursing students' opinions about health, spirituality and religiosity.

a Enfermeira. Graduada pela Faculdade de Medicina de Marília (FAMEMA). Membro da Sociedade Científica de Saúde e Espiritualidade da Faculdade de Medicina de Marília (SOCISE-FAMEMA). Marília, SP, Brasil.

b Acadêmica de Enfermagem da FAMEMA. Membro da SOCISE-FAMEMA. Marília, SP, Brasil.

c Acadêmica de Enfermagem da FAMEMA. Marília, SP, Brasil.

d Enfermeira. Doutora em Enfermagem pela Escola de Enfermagem de Ribeirão Preto da Universidade de São Paulo. Professora Titular da FAMEMA. Membro da SOCISE-FAMEMA. Marília, SP, Brasil.

e Médico. Professor adjunto do departamento de clínica médica da Faculdade de Medicina da Universidade Federal de Juiz de Fora, MG, Brasil. Doutor em ciências pelo departamento de Neurologia/neurociências pela Universidade Federal de São Paulo. Colaborador de pesquisa da Associação Médico-Espírita do Brasil. Orientador da SOCISE-FAMEMA. São Paulo, SP, Brasil. 


\section{INTRODUÇÃO}

A Enfermagem moderna tem focado cada vez mais sua atenção na humanização do cuidado em saúde e, em uma visão mais ampla da interface saúde-doença. Falar sobre os aspectos que transcendem a dimensão biológica do paciente, como a espiritualidade e a religiosidade, é importar-se com as experiências e vivências do ser humano ${ }^{(1)}$.

Um número crescente de estudos aponta a influência da espiritualidade na prática clínica em diversas situações ${ }^{(2)}$, incluindo menor prevalência de depressão, menores níveis pressóricos, menores complicações pós-cirúrgicas ${ }^{(3)}$ e maior bem-estar psicológico, incluindo satisfação com a vida, felicidade, afeto positivo e moral elevada ${ }^{(4)}$.

Da mesma forma, diversos estudos têm mostrado que os pacientes desejam receber esse tipo de suporte, variando de 33 a $94 \%$ nos estudos internacionais $^{(5)}$ e 79 a $87 \%$ nos estudos brasilei$\operatorname{ros}^{(3,6)}$, dependendo do tipo de atendimento, local de atuação e contexto clínico.

Apesar do grande número de evidências, o conceito de espiritualidade ainda não é consensual. A espiritualidade pode ser definida como uma busca pessoal para entender questões relacionadas o fim da vida, ao seu sentido, sobre as relações com o sagrado ou transcendente que, pode ou não, levar ao desenvolvimento de práticas religiosas ou formações de comunidades religiosas ${ }^{(5)}$. Já religiosidade é o quanto um indivíduo acredita, segue e pratica uma religião. Na religiosidade intrínseca ${ }^{(7)}$ a religião tem um lugar central na vida do indivíduo, é seu bem maior. Na religiosidade extrínseca a religião é um meio utilizado para obter outros fins, como consolo, sociabilidade, distração e status.

$\mathrm{Na}$ compreensão destes sentidos, evidencia-se um aumento no reconhecimento de que a espiritualidade é um aspecto fundamental da assistência de Enfermagem. No entanto, muitos profissionais ainda se sentem hesitantes e com pouca confiança para abordar estes aspectos, abarcados pela falta de inclusão adequada dessa temática durante o processo de formação acadêmica ${ }^{(8)}$. Sem modelos bem estruturados de avaliação e treinamento dos profissionais em formação, torna-se dificultada a introdução desse suporte na prática clínica diária, já tão sobrecarregada com funções administrativas e pelo pouco tempo disponível.

Seguindo essa tendência, a NANDA International (NANDA I), ao atentar para o cuidado espiritual nos diagnósticos de Enfermagem, fornece estímulo à importância de um estudo formal acerca da temática Saúde e Espiritualidade na $\operatorname{graduação~}^{(9)}$. De acordo com recente estudo ${ }^{(10)}$, muitos alunos do curso de Enfermagem acreditam que a espiritualidade influencia na saúde de seus pacientes e no próprio atendimento por parte do enfermeiro e, apesar de a maioria desejar abordar esse aspecto, poucos se julgam preparados para tal. Notavelmente, mais de $90 \%$ dos alunos avaliados por esse estudo, acreditam que a universidade não proporciona todas as informações necessárias para este preparo.

Mesmo que a atuação por parte da Enfermagem no respeito ao cuidado espiritual seja legítima, podendo ser oferecida até mesmo durante o processo de trabalho ${ }^{(11)}$, o ensino da temática durante a graduação teria um papel importante para a vida do estudante no desenvolvimento dos valores do cuidado holístico $^{(12)}$. Os alunos tendem a espelhar-se em seus professores e, sem uma abordagem mais clara do assunto, acabam julgando desnecessária ou ainda fora do âmbito de seu trabalho.

Dessa forma, compreender o que pensam os estudantes de Enfermagem e como agem perante a relação espiritualidade, religiosidade e saúde, possibilitaria reflexões acerca do preparo fornecido na graduação para a construção de profissionais que exercem um cuidado mais integral, identificação das lacunas e proposição de formas para a implementação do assunto no currículo.

Sendo assim, o presente estudo objetivou identificar as opiniões dos estudantes de Enfermagem em relação à interface entre saúde, espiritualidade e religiosidade e as informações fornecidas durante o período da formação acadêmica

\section{MÉTODO}

Foi realizado um estudo exploratório, descritivo, transversal e de natureza quantitativa no Curso de Graduação de Enfermagem da Faculdade de Medicina de Marília (FAMEMA), situada em uma cidade do interior do estado de São Paulo. A população elegível do estudo foi composta por todos os discentes regularmente matriculados no Curso de Graduação em Enfermagem da FAMEMA durante o período letivo de 2011 , perfazendo um total de 146 estudantes. Aqueles que realizaram trancamento da matrícula 
ou que não foram localizados foram excluídos da pesquisa, bem como os pesquisadores acadêmicos que participaram do estudo.

A coleta de dados foi realizada pelos pesquisadores entre os meses de maio a outubro de 2011. Utilizou-se um questionário padrão, auto-aplicável, entregue a todos os participantes do estudo, mediante autorização institucional para aplicação do instrumento em momentos oportunos, que não interferissem nas atividades acadêmicas dos sujeitos. Apesar dos estudantes terem sido orientados a preencher o instrumento presencialmente, alguns interessados em participar da pesquisa e que não conseguiriam fazê-lo no momento específico, foram autorizados a preencher em um segundo momento.

O instrumento utilizado foi adaptado de um estudo multicêntrico envolvendo acadêmicos de medicina de diversas faculdades do Brasil, coordenado pela Universidade Federal de São Paulo - UNIFESP ${ }^{(19)}$. As questões foram divididas em:

- Dados sócio-demográficos: gênero, idade, ano de graduação, etnia e renda familiar;

- Prática clínica, o paciente e a espiritualidade: avaliação do conceito de espiritualidade apontado pelos discentes, conhecimentos sobre a relação entre a espiritualidade e a saúde e como este contex to pode estar inserido na prática clínica do enfermeiro;

- A formação acadêmica e o tema espiritualidade: conhecimento e opinião sobre a forma pela qual a universidade aborda o tema durante a graduação e como poderia ser oferecido o preparo para trabalhar com o assunto;

- Dimensão de religiosidade: foram avaliados diferentes aspectos da espiritualidade e da religiosidade do participante através da afiliação religiosa e da utilização do Índice de Religiosidade de Duke (Duke Religious Index - DUREL). Esta escala foi validada para o Brasil $^{(14)}$ e possui cinco questões (tipo Likert) que avaliam três das dimensões de religiosidade que mais se relacionam com desfechos em saúde: organizacional, não organizacional e intrínseca.

Os dados obtidos foram tabulados em Excel e analisados por meio do programa SPSS 17.0. Foram realizadas tabelas de contingência e medidas resumo do perfil, opiniões e influência na formação acadêmica dos participantes.

O projeto foi submetido ao Comitê de Ética em Pesquisa Envolvendo Seres Humanos da Faculdade de Medicina de Marília, aprovado sob o n ${ }^{\circ}$ 557/ 10. Todos participantes assinaram o Termo de Consentimento Livre e Esclarecido.

\section{RESULTADOS}

Participaram do estudo 120 dos 146 discentes do Curso de Graduação em Enfermagem, totalizando uma taxa de resposta ao questionário de $82,19 \%$. No geral, todos os anos letivos foram contemplados com mais de $75 \%$ de respostas sendo: 36 (78,26\%) alunos pertencentes ao $1^{\circ}$ ano, $22(88 \%)$ ao $2^{\circ}$ ano, $30(90,90 \%)$ ao $3^{\circ}$ ano e $32(76,19 \%)$ estudantes do $4^{\circ}$ ano.

\section{Características sócio-demográficas dos participantes}

Os sujeitos eram constituídos, em sua maioria, do gênero feminino (90\%), etnia branca (76,3\%) e renda familiar abaixo de sete salários mínimos $(67,9 \%)$. Com relação à idade dos estudantes, esta variou entre 17 e 34 anos, com predominância entre 20 e 25 anos $(65,2 \%)$ e média de $20,70 \pm$ 3,29 anos.

\section{Crenças e religiosidade}

A maioria dos participantes (87,9\%) possuía alguma afiliação religiosa e 67,8\% consideravam-se pessoas "moderadamente religiosas" (pelo auto-relato de religiosidade, variando de "não religioso", "pouco religioso", "moderadamente religioso" e "muito religioso"). Cerca de 32,8\% dos participantes afirmaram frequentar um serviço religioso pelo uma vez por semana e $43,7 \%$ dos estudantes dedicavam-se, diariamente, a atividades religiosas individuais como preces, meditações e leituras religiosas.

$\mathrm{Na}$ avaliação da religiosidade intrínseca, obtida através da Escala de Religiosidade, os participantes obtiveram média de $5,57 \pm 2,10$ pontos, indicando uma grande religiosidade intrínseca. As opções de resposta desta dimensão foram oferecidas em direção decrescente, onde o escore mais alto (15) é relacionado à menor religiosidade intrínseca e o 
escore mais baixo (3) é relacionado a maior religiosidade intrínseca.

A unanimidade dos participantes apontou acreditar em Deus; 92,4\% acreditavam que o ser humano é composto de um corpo e uma alma/espírito e $80,8 \%$ acreditavam que esta alma esta ainda preserva-se viva após a morte física.

\section{Conceito de espiritualidade}

Quanto ao conceito de espiritualidade atribuído pelos discentes, utilizamos uma questão fechada de múltipla escolha em que era possível assinalar mais de uma alternativa. Como resultado, a maioria dos participantes $(61,7 \%)$ apontou o conceito desse termo como "crença e relação com Deus/religiosidade", seguido por "busca de sentido e significado para a vida humana" (40,8\%), "crença em algo transcendente à matéria" (26,7\%), "crença na existência da alma e na vida após a morte" $(24,2 \%)$ e "postura ética e humanística" (13,3\%).

\section{Prática clínica, o paciente e a espiritualidade}

A maioria dos estudantes considerava que a religião/espiritualidade exercia muita influência na saúde dos pacientes (84,9\% muito/extremamente; $13,4 \%$ mais ou menos; $1,7 \%$ pouco e $0,0 \%$ muito pouco ou nada). Para $66,7 \%$ dos participantes, esta influência era geralmente positiva, para 30,0\% era igualmente positiva e negativa e para $3,3 \%$ a influência era negativa ou não existia.

Os participantes ainda consideraram pertinente a abordagem dos aspectos religiosos/espirituais dos pacientes na prática clínica e frequentemente tinham vontade de realizar esta abordagem $(41,5 \%)$, sendo que a maioria dos alunos $(71,4 \%)$ já perguntou sobre a religião/espiritualidade dos pacientes (Tabela 1).

Entretanto, quando questionados se sentiam preparados para abordar os aspectos religiosos/ espirituais com os pacientes, pouco mais da metade dos discentes (50,8\%) afirmaram estar "moderadamente preparados" e 32,5\% disseram estar "pouco preparados". Entre os principais motivos apontados, encontrou-se: "falta de conhecimento" $(34,8 \%)$, "falta de treinamento" $(39,1 \%)$, "falta de tempo" $(26,1 \%)$, "desconforto com o tema" (11,3\%), "medo de impor pontos de vista religiosos aos pacientes" (60,9\%), "não faz parte do meu trabalho" $(1,7 \%)$, "medo de ofender os pacientes" (36,5\%), "medo de que meus colegas não aprovem" $(8,7 \%)$ e "outros motivos" $(5,2 \%)$ (Tabela 1 ).

\section{A formação acadêmica e o tema "saúde e espiritualidade"}

$\mathrm{Na}$ formação universitária atual, grande parte dos discentes avaliou negativamente a abordagem de temas sobre crenças religiosas ou espirituais nas atividades curriculares. Apesar do fato, os alunos apontaram que os docentes do $1^{\circ}$ ano da graduação eram os que mais abordavam tais aspectos (Tabela 2).

Em relação ao ofertado pela faculdade, 91,6\% dos alunos acreditavam ser insuficientes as informações fornecidas para que pudessem abordar as crenças religiosas ou espirituais com os pacientes.

No que tange à formação dos discentes sobre o tema, $78,0 \%$ dos participantes referiu não ter participado de atividades que auxiliassem neste processo, no entanto, afirmaram que gostariam de participar.

Quando questionados em relação à forma com que buscavam conhecimento sobre temas entre saúde e espiritualidade, os alunos relataram ser através de ensinamentos dentro da própria religião $(54,6 \%)$, leitura de livros $(17,6 \%)$, leitura de artigos científicos (16\%), palestras $(9,2 \%)$, docentes da faculdade $(4,2 \%)$ e $27,7 \%$ não buscavam conhecimentos.

Ao serem questionados se acreditavam que temas relacionados à saúde e espiritualidade deveriam fazer parte dos currículos de Enfermagem, a maioria dos participantes respondeu afirmativamente $(83,8 \%)$ e grande parte $(73,1 \%)$ mencionou que não tiveram mudanças de suas crenças ou condutas em relação à religiosidade/espiritualidade após a entrada na faculdade.

\section{DISCUSSÃO}

A amostra do presente estudo teve predominância do gênero feminino (90\%), o que corrobora com dados nacionais de que o sexo feminino exerce predominância nos cursos de graduação em Enfermagem ${ }^{(10)}$.

A maioria dos participantes considerou como positiva a influência que a religião e a espiritualidade exercem na saúde dos pacientes. De fato, estudos evidenciam que a prática religiosa está 
Tabela 1 - Abordagem da religião/espiritualidade pelo discente de Enfermagem. Marília, SP, 2011.

\begin{tabular}{|c|c|c|c|}
\hline & & $\mathbf{N}$ & $\%$ \\
\hline \multirow{6}{*}{$\begin{array}{l}\text { O quanto você acha } \\
\text { pertinente abordar aspectos } \\
\text { religiosos/espirituais com os } \\
\text { pacientes? }\end{array}$} & Muitíssimo pertinente & 17 & 14,3 \\
\hline & Muito pertinente & 53 & 44,5 \\
\hline & Moderadamente pertinente & 39 & 32,8 \\
\hline & Pouco pertinente & 6 & 5,0 \\
\hline & Nada pertinente & 4 & 3,4 \\
\hline & Total & 119 & 100,0 \\
\hline \multirow{4}{*}{$\begin{array}{l}\text { Você sente vontade } \\
\text { de abordar o tema fé/ } \\
\text { espiritualidade com os } \\
\text { pacientes? }\end{array}$} & Sim, raramente & 44 & 37,3 \\
\hline & Sim, freqüentemente & 49 & 41,5 \\
\hline & Não & 25 & 21,2 \\
\hline & Total & 118 & 100,0 \\
\hline \multirow{4}{*}{$\begin{array}{l}\text { Você alguma vez já } \\
\text { perguntou sobre a religião/ } \\
\text { espiritualidade dos } \\
\text { pacientes? }\end{array}$} & Sim & 75 & 71,4 \\
\hline & Não & 21 & 20,0 \\
\hline & Não se aplica, eu não vejo pacientes & 9 & 8,6 \\
\hline & Total & 105 & 100,0 \\
\hline \multirow{7}{*}{$\begin{array}{l}\text { O quanto você se considera } \\
\text { preparado para abordar } \\
\text { aspectos religiosos/ } \\
\text { espirituais com os pacientes? }\end{array}$} & Muitíssimo preparado & $\mathrm{O}$ & $\mathrm{O}$ \\
\hline & Muito preparado & 10 & 8,3 \\
\hline & Moderadamente preparado & 61 & 50,8 \\
\hline & Pouco preparado & 39 & 32,5 \\
\hline & Nada preparado & 7 & 5,8 \\
\hline & Não se aplica & 3 & 2,5 \\
\hline & Total & 120 & 100,0 \\
\hline \multirow{10}{*}{$\begin{array}{l}\text { Alguma das afirmações } \\
\text { seguintes desencoraja } \\
\text { você a discutir religião/ } \\
\text { espiritualidade com os } \\
\text { pacientes?* }\end{array}$} & Falta de conhecimento & 40 & 34,8 \\
\hline & Falta de treinamento & 45 & 39,1 \\
\hline & Falta de tempo & 30 & 26,1 \\
\hline & Desconforto com o tema & 13 & 11,3 \\
\hline & $\begin{array}{l}\text { Medo de impor pontos de vista religiosos } \\
\text { aos pacientes }\end{array}$ & 70 & 60,9 \\
\hline & $\begin{array}{l}\text { Conhecimento sobre religião não é } \\
\text { relevante no cuidado }\end{array}$ & $\mathrm{O}$ & 0 \\
\hline & Não faz parte do meu trabalho & 2 & 1,7 \\
\hline & Medo de ofender os pacientes & 42 & 36,5 \\
\hline & Medo de que meus colegas não aprovem & 10 & 8,7 \\
\hline & Outros & 6 & 5,2 \\
\hline
\end{tabular}

*Questão em que era possível assinalar mais de uma alternativa Fonte: dados dos autores

relacionada com repercussões no eixo psiconeuroimunológico(5), além de ser fator de prevenção para o desenvolvimento de doenças em populações previamente sadias, relacionando-se à eventual redução de óbito e ao impacto de diversas doenças ${ }^{(3,}$ 15). Entretanto, poucos estudantes lembraram-se dos efeitos negativos da religiosidade como, por exemplo, o sofrimento religioso que levaria a uma maior mortalidade e piores desfechos clínicos ${ }^{(16)}$. Esse achado remete-nos a visão distorcida de que a religiosidade só produz efeitos positivos na saúde do paciente, subjulgando os diversos estudos sobre os aspectos negativos das crenças religiosas ${ }^{(17)}$. A abordagem do paciente deve ser criteriosa 
Tabela 2 - Abordagem da religião/espiritualidade na formação acadêmica de enfermagem. Marília, SP, 2011.

\begin{tabular}{|c|c|c|c|}
\hline & & $\mathbf{N}$ & $\%$ \\
\hline \multirow{6}{*}{$\begin{array}{l}\text { Os docentes já abordoram temas sobre } \\
\text { crenças religiosas ou espirituais nas } \\
\text { atividades curriculares? }\end{array}$} & Nunca & 52 & 48,1 \\
\hline & Raramente & 29 & 26,9 \\
\hline & Algumas vezes & 26 & 24,1 \\
\hline & Comumente & 1 & 0,9 \\
\hline & Sempre & 0 & 0 \\
\hline & Total & 108 & 100,0 \\
\hline \multirow{4}{*}{ Em qual ano da graduação?* } & $1^{\circ}$ ano & 42 & 59,2 \\
\hline & $2^{\circ}$ ano & 13 & 18,3 \\
\hline & $3^{\mathrm{o}}$ ano & 13 & 18,3 \\
\hline & $4^{o}$ ano & 3 & 4,2 \\
\hline \multirow{7}{*}{$\begin{array}{l}\text { A formação universitária atual fornece } \\
\text { informações suficientes para que os } \\
\text { acadêmicos consigam abordar as crenças } \\
\text { religiosas ou espirituais dos pacientes? }\end{array}$} & Nem um pouco & 54 & 45,8 \\
\hline & Um pouco & 37 & 31,4 \\
\hline & Mais ou menos & 17 & 14,4 \\
\hline & Bastante & 1 & 0,8 \\
\hline & Muitíssimo & 1 & 0,8 \\
\hline & Não tenho opinião formada & 8 & 6,8 \\
\hline & Total & 118 & 100,0 \\
\hline \multirow{4}{*}{$\begin{array}{l}\text { Você já participou de alguma atividade } \\
\text { de formação sobre a relação "Saúde e } \\
\text { Espiritualidade"? }\end{array}$} & Sim & 15 & 12,7 \\
\hline & Não, mas gostaria de participar & 92 & 78,0 \\
\hline & Não e não gostaria de participar & 11 & 9,3 \\
\hline & Total & 118 & 100,0 \\
\hline \multirow{6}{*}{$\begin{array}{l}\text { De que forma você busca conhecimentos } \\
\text { sobre o tema "Saúde e Espiritualidade"?* }\end{array}$} & Assisto palestras que abordam o tema & 11 & 9,2 \\
\hline & Leio livros & 21 & 17,6 \\
\hline & Leio artigos científicos & 19 & 16,0 \\
\hline & $\begin{array}{l}\text { Através dos docentes de minha } \\
\text { faculdade }\end{array}$ & 5 & 4,2 \\
\hline & Dentro da minha própria religião & 66 & 55,5 \\
\hline & Eu não busco conhecimentos & 33 & 27,7 \\
\hline
\end{tabular}

*Questão respondida em caso afirmativo da pergunta anterior, sendo possível assinalar mais de uma alternativa Fonte: dados dos autores

e baseada em treinamento prévio, de forma a não impor crenças ou fazer julgamentos.

Apesar dos alunos terem exprimido a vontade de realizar a abordagem dos aspectos religiosos e espirituais dos pacientes na prática clínica, eles acreditavam que ainda não possuíam segurança para esta prática e que os conhecimentos apreendidos dentro da graduação eram insuficientes para o desenvolvimento desta competência. Em geral, a abordagem da espiritualidade durante a formação universitária não é suficientemente profunda, principalmente no que se refere às discussões teóricas ${ }^{(18)}$ e a própria parte prática, necessárias ao preparo do aluno ${ }^{(10)}$. Muitas vezes, a atenção dada ao tema ocorre através de conversas e orações por meio da participação de capelães, porém com pouca aplicabilidade clínica e pouco baseada em evidências. 
Nossos dados são correlatos a estudos prévios. Em estudo realizado com 30 enfermeiros de um hospital de grande porte, os resultados evidenciaram que, apesar de a maioria dos sujeitos terem respondido afirmativamente sobre a importância em oferecer assistência espiritual ao paciente, $67 \%$ dos entrevistados referiu não ter recebido uma formação profissional para prestar assistência espiritual ao paciente no curso de graduação $^{(9)}$. Da mesma forma, estudo recente ${ }^{(10)}$ demonstrou que $98 \%$ dos participantes relataram que a formação universitária não trazia informações suficientes sobre o tema "Saúde e espiritualidade".

A inserção de disciplinas ou ainda módulos baseados em problemas ou metodologia ativa poderiam, de certa forma, auxiliar no desenvolvimento acadêmico frente a essa área. Porém, a capacitação dos docentes, principalmente aqueles que atuam nos últimos semestres de curso, torna-se essencial no contex to da enfermagem, haja visto que o aluno é mais exposto a esse tipo de situação quando está nas atividades práticas do estágio.

A falta de preparo do aluno encontrada em nosso estudo parece interferir de forma negativa na possibilidade de uma abordagem mais ampla do assunto no campo da Enfermagem. A principal barreira identificada refere-se ao medo de impor pontos de vista religiosos aos pacientes. Esse resultado é similar ao encontrado em estudo realizado com 118 discentes do curso de Enfermagem, que também apontaram o medo de impor suas crenças como o principal motivo ${ }^{(10)}$.

Este receio pode ser justificado por alguns fatores: falta de treinamento e embasamento científico adequados para uma abordagem mais ecumênica e mais centrada no paciente e desconhecimento das diferenças inerentes aos termos religiosidade e espiritualidade. Como colocado anteriormente, a espiritualidade está relacionada à busca pessoal pelo entendimento de respostas a questões sobre a vida, seu significado e relações com o sagrado e transcendente, que pode ou não estar relacionada a propostas de uma determinada religião( ${ }^{(5)}$. Este conceito diverge do atribuídos pelos discentes do presente estudo, em que a maioria definiu espiritualidade como relação vinculada à religiosidade.

Outro motivo apontado pelos estudantes para a não abordagem da dimensão religiosa e espiritual do pacientes refere-se ao medo de ofender os pacientes. É importante destacar que o Código de Ética dos Profissionais de Enfermagem, no artigo $6^{\circ}$, expõe que o enfermeiro deve "fundamentar suas relações no direito, na prudência, no respeito, na solidariedade e na diversidade de opinião e posição ideológica" ${ }^{(19)}$. No entanto, equilibrar o direito fundamental à vida de um lado e os direitos fundamentais à intimidade, à privacidade e à liberdade por motivo de convicção religiosa de outro, torna-se uma difícil tarefa ao considerar a formação profissional baseada na racionalidade biomédica de muitos enfermeiros. Sendo assim, a integração entre espiritualidade, fé, religiosidade e ética torna-se uma nova faceta do cuidado ${ }^{(18)}$.

Vários alunos referiram acreditar que a religião/espiritualidade dos enfermeiros interfere com grande intensidade $(42,4 \%)$ no entendimento do processo saúde-doença e na relação profissional-paciente. Vale ressaltar que, segundo alguns autores $^{(18)}$, a raiz religiosa está tão imbricada na memória coletiva que, mesmo em instituições que não se declaram religiosas, os pressupostos cristãos se mantêm presentes e com vitalidade, o que também pode ser corroborado com a unanimidade dos sujeitos do presente estudo acreditarem em Deus.

Quando questionados em relação à forma com que buscavam conhecimento sobre temas entre saúde e espiritualidade, a maioria dos alunos relatou ser através de ensinamentos dentro da própria religião. O que pode ocorrer é o desconhecimento por parte dos alunos da área de saúde das evidências na literatura científica sobre a ligação entre espiritualidade, saúde física ${ }^{(3)}$ e saúde mental ${ }^{(4)}$, mesmo que, ao inserir os unitermos "religiosity", "religion", "spiritual" e "spirituality" na base de dados eletrônica MEDLINE, encontra-se cerca de 40 mil $\operatorname{artigos}^{(2)}$.

Esse achado reforça o desconhecimento do estudante perante ao assunto e a busca não baseada em evidências. Os conhecimentos e inserção religiosos podem ser importantes para o desenvolvimento espiritual/religiosos dos enfermeiros mas, podem não contemplar todas as informações necessárias para uma correta abordagem da espiritualidade no cuidado em saúde. Este fato deixa clara a necessidade de orientação aos alunos a respeito das informações disponíveis para o aprimoramento da formação do profissional de Enfermagem, de forma a entenderem que esse tipo de abordagem fará parte de sua profissão como enfermeiro. 


\section{CONCLUSÃO}

No presente estudo, os estudantes do curso de Enfermagem consideraram que a abordagem da espiritualidade dos pacientes exercia influência na prática clínica. Apesar da maioria já ter perguntado sobre a religião ou espiritualidade dos pacientes, poucos se sentiam adequadamente preparados e tiveram treinamento sobre o assunto. As principais barreiras para essa abordagem foram o medo de impor pontos de vista religiosos aos pacientes e o medo de ofender os pacientes.

Quanto a abordagem da religião/espiritualidade na formação acadêmica, os alunos relataram que as informações fornecidas pela faculdade eram insuficientes e que buscavam conhecimento sobre o assunto dentro da própria religião em detrimento a leitura de livros e artigos científicos.

Algumas limitações devem ser apontadas na análise de nossos resultados. Primeiro, os dados são provenientes de uma única faculdade de enfermagem. Pesquisas adicionais em diferentes instituições de ensino são necessárias para que um perfil mais amplo dos estudantes de Enfermagem seja caracterizado. Segundo, por tratar-se de um estudo transversal, não é possível entender como a formação acadêmica modificaria as opiniões do aluno frente ao assunto no decorrer da graduação.

Esses dados nos remetem as lacunas na inserção do assunto e no treinamento proporcionado durante a formação acadêmica. Outro importante aspecto a ser refletido por esses achados relaciona-se às grandes transformações humanas envolvidas na globalização, que tem instaurado uma profunda crise do "humanismo", no qual deparamo-nos diuturnamente com ambientes de altos padrões tecno-científicos, mas carentes de uma abordagem mais humanizada ${ }^{(20)}$.

Conclui-se a necessidade da implementação de cenários de aprendizagem durante a formação acadêmica que promovam a competência no estudante para realizar o cuidado espiritual.

\section{REFERÊNCIAS}

1 Pessini L. A espiritualidade interpretada pelas ciências e pela saúde. Mundo Saúde. 2007;31(2):18795.
2 Moreira-Almeida A. O crescente impacto das publicações em espiritualidade e saúde eo papel da Revista de Psiquiatria Clínica. Rev Psiquiatr Clín. 2010;37(2):41-2.

3 Lucchetti G, Lucchetti AGL, Badan-Neto AM, Peres PT, Peres MF, Moreira-Almeida A, et al. Religiousness affects mental health, pain and quality of life in older people in an outpatient rehabilitation setting. J Rehabil Med. 2011;43(4):316-22.

4 Bonelli RM, Koenig HG. Mental disorders, religion and spirituality 1990 to 2010 : a systematic evidence-based review. J Relig Health. 2013;52(2):657-73.

5 Koenig H, King D, Carson VB. Handbook of religion and health. $2^{\text {a }}$ ed. New York: Oxford University Press; 2012.

6 Lucchetti G, Almeida LGC, Lucchetti ALG. Religiousness, mental health, and quality of life in brazilian dialysis patients. Hemodial Int. 2012;16(1):89-94.

7 Donahue MJ. Intrinsic and extrinsic religiousness: review and meta-analysis. J Pers Soc Psychol. 1985;48(2):400-19.

8 McSherry W, Jamieson S. An online survey of nurses' perceptions of spirituality and spiritual care. J Clin Nurs. 2011;20(11-12):1757-67.

9 Pedrão RB, Beresin R. O enfermeiro frente à questão da espiritualidade. Rev Einstein. 2010;8:86-91.

10 Tomasso CdS, Beltrame IL, Lucchetti G. Knowledge and attitudes of nursing professors and students concerning the interface between spirituality, religiosity and health. Rev Latinoam Enferm. 2011;19(5):1205-13.

11 Smith AR. Using the synergy model to provide spiritual nursing care in critical care settings. Crit Care Nurse. 2006;26(4):41-7.

12 Wu L-F, Lin LY. Exploration of clinical nurses' perceptions of spirituality and spiritual care. J Nurs Res. $2011 ; 19(4): 250-6$.

13 Lucchetti G, de Oliveira LR, Granero Lucchetti AL, Leite JR. Spirituality in medical education: new initiatives in Brazil. Clin Teach. 2011;8(3):213.

14 Lucchetti G, Lucchetti ALG, Peres MF, Leão FC, Moreira-Almeida A, Koenig HG. Validation of the duke religion index: DUREL (portuguese version). J Relig health. 2012;51(2):579-86. 
15 Moreira-Almeida A, Lotufo Neto F, Koenig HG Religiousness and mental health: a review. Rev Bras Psiquiatr. 2006;28(3):242-50.

16 Pargament KI, Koenig HG, Tarakeshwar N, Hahn J. Religious struggle as a predictor of mortality among medically ill elderly patients: a 2-year longitudinal study. Arch Intern Med. 2001;161(15):1881-85.

17 Chaves EdCL, Carvalho E, Hass VJ. Validação do diagnóstico de enfermagem Angústia Espiritual: análise por especialistas. Acta Paul Enferm. 2010;23(2):264-70.
18 Gussi MA, JLG D. Religião e espiritualidade no ensino e assistência de enfermagem. Rev Bras Enferm. 2008;61(3):377-84.

19 Conselho Federal de Enfermagem(BR). Resolução n. 311, de 8 de fevereiro de 2007: aprova a reformulação do Código de Ética dos Profissionais de Enfermagem [Internet]. Brasília(DF); 2007 [citado 2013 Jan 01]. Disponível em: http://site.portalcofen.gov.br/node/4345.

20 Barchifontaine CP. Espiritualidade e comunicação na saúde: fundamentação conceitual. Mundo Saúde. 2010;34(4):475-82.

\author{
Endereço do autor / Dirección del autor / \\ Author's address \\ Giancarlo Lucchetti \\ Rua Dona Elisa, 150, ap. 153 B, Barra Funda \\ 01155-030, São Paulo, SP \\ E-mail: g.lucchetti@yahoo.com.br
}

Recebido em: 04.09.2013

Aprovado em: 27.11.2013 